\title{
Analysis of wavelet based denoising methods applied to measured lightning electric fields
}

\begin{abstract}
This paper describes the results of a wavelet based noise reduction procedure applied to three lightning electric field signals recorded in Bogotá-Colombia, Colombo-Sri Lanka and Toronto-Canada. In general, the evaluated signals were affected by three different noise sources. These are the analog-to-digital converter (ADC), the electronic circuit and the environmental noise.

The effectiveness of the wavelet based denoised technique was demonstrated in a previous work, and this paper advances the technique by using an adaptive wavelet approach to automate the procedure, including the proper selection of the thresholds.

From the wavelet transform, the main noise effects in the electric field signatures were reduced and then several lightning electric field temporal characteristics were analyzed. In all cases the initial peak occurs during the first few microseconds. The maximum electric field, the maximum electric field derivative, and the electric field rise-time were also evaluated.
\end{abstract}

